\title{
Transition Metal Chemistry; past, present and future
}

\author{
Marcus C. Durrant ${ }^{1}$
}

Published online: 28 January 2019

(c) Springer Nature Switzerland AG 2019

This year of 2019 marks the thirtieth anniversary of my involvement with Transition Metal Chemistry (TMC). In 1989, I started work at the Nitrogen Fixation Laboratory, a government-funded research institute on the University of Sussex campus. I had completed my PhD at Sussex a few years earlier, under the joint supervision of Harry Kroto and David Walton. When I met up with David for a chat, he suggested that I might like to join the TMC editorial team. It was always a pleasure to work with David, so I was very happy to do so.

At that time, many aspects of scientific publishing were very different to those of today. This was just before the electronic revolution which would enable online submission, refereeing and publication of manuscripts. Papers had to be typed (in triplicate) by hand, usually by a secretary who had to decipher the academic's handwritten notes. The paper copies were then mailed into the office, with referees also mailing their reports back to the office on carbon copy sheets. David had a complete shelf of TMC box files in his office, each full of manuscripts at various stages of the refereeing process. Everything was kept meticulously organized by his secretary Jean Hudson, with the help of oversized paper clips and a selection of rubber stamps, from the initial 'Received on [date]' stamp to the dreaded 'Rejected' stamp. Meanwhile, in the university library, shelf after shelf would gradually be filled with bound copies of the various journals. Many older chemists will remember spending hours combing through volume after volume, searching for that one piece of critical information. For me, the contemplation of row after row of journals in those old paper-based libraries always brought a powerful thought to mind, namely that science is much greater than a single human lifespan. You could pick up the very latest, unbound issues of a journal on the right-hand end of a shelf, but a few paces to the left would bring you back in time by a century or more, to the

Marcus C. Durrant

mcd.tmc@zoho.com

1 Northumbria University, Newcastle-upon-Tyne, UK work of previous generations of chemists. As authors, we each get to contribute a few pages, no more, to that vast and ever-expanding stock of human knowledge, before we too, take our place in history. Those few pages will remain, perhaps carrying a little of the essence of who we were as professional scientists. In one or two hundred years' time, someone may revisit our work and read our carefully written prose, which no doubt by then will seem archaic in style, perhaps even pausing to catch an imaginary glimpse of the author in their mind's eye. It is a humbling thought that should encourage us all to make our contributions the best that they can be.

Another big difference was the composition of staff at most university faculties. When I was an undergraduate, each and every one of the chemistry faculty was a middle class or even faintly aristocratic white man, just as the mechanical and glassblowing workshops were generally staffed by working-class men, and the departmental secretaries were all women. Although much has changed over the decades, the culture of scientific publishing that we have today still has its roots in that bygone age. In particular, scientific publishing grew out of the ethos of the professional scientific societies of the nineteenth century. The publication of papers in the learned journals of these societies was governed by what might be termed a culture of 'gentlemen's agreement'. With much smaller research communities, referees were happy to consider one another's papers, as a service to the scientific community and a useful way of keeping up with their peers. In return, authors were careful to submit their contributions to appropriate journals, avoiding large numbers of rejections. University libraries could afford to stock all the major journals in hardcopy form. The overall pace of research was a good deal slower, such that academics had a lot more time to think about what they were doing, and why they were doing it, as well as polishing their manuscripts. There was also much less pressure to publish in the first place; I remember Harry Kroto once remarking that, when an academic was faced with imminent death, they would not see their lives flashing before them, but rather their unpublished papers! 
The upshot of this historical background, as we make our way through the twenty-first century, is that the system of academic publishing that we have all inherited is far from ideal. As with many other aspects of science, the need for post hoc organization has thrown up many anomalies and difficulties. Perhaps, the most obvious issue is the need for many authors to acquire a lengthy list of publications in order to advance their careers. Although this dovetails with the need of publishers to find enough content for their journals, it leads many authors into a continual search to find the smallest, most incremental portion of their research that has a sporting chance of meeting with the approval of referees. This in turn creates a massive overhead of work for editors and referees, who spend much of their time sifting through the deluge of papers entering the system and discarding those that fail the minimal content test. Furthermore, because electronic submission is so easy, it is not unusual for the same paper to be rejected again and again by different journals. This probably explains why the TMC rejection rate has remained between 71 and $81 \%$ over the last decade. This futile cycling of manuscripts around the system suggests that the 'gentlemen's agreement' has outlived its value and needs a fundamental rethink. Nevertheless, encouragingly, at TMC we have increased our average pages-per-paper from 6.6 in 2007 to 9.1 in 2017. This has been accompanied by an increase in the journal's impact factor from 0.84 to 1.26 over the same period. Although we cannot be sure of a correlation between these figures, the implication that more substantial papers are of greater value to the scientific community seems reasonable.

How has the chemistry reported in TMC changed over the last 30 years? Perhaps, the biggest single difference has come from the far greater ease with which X-ray crystal structures can now be obtained. Data collection and processing have both become very much faster, such that work which might have taken weeks or even months now takes days. This is reflected in the growth of the Cambridge Crystallographic Data Centre database (CCDC); in 1989, the total number of CCDC entries stood at 87,424 ; it is now forecast to pass one million structures by July 2019 [1], with about 50,000 new structures currently added every year. For TMC, this means that many of our published papers are now supported by at least one X-ray crystal structure. This is especially important in coordination chemistry, where other methods such as physico-chemical analysis and spectroscopy are rarely adequate for the definitive characterization of chemical structures. The good news for TMC is that we now reject far fewer papers based on 'inadequate characterization'. At one time, I kept a list of 'implausible structural motifs' that occurred repeatedly and were considered sufficient grounds for a rejection. Three of these are shown in Scheme 1; readers might like to spot the deliberate mistakes (the answers are given below).

In principle, the underpinning of contemporary transition metal chemistry with supporting crystal structures allows for systematic structure-function analysis. While this is possible in some areas, such as catalysis and the development of novel luminescent complexes, it remains a daunting challenge in others. For example, metal-organic frameworks show such a variety of structures that their prediction from first principles generally remains an aspiration rather than a realistic goal. In metallodrug discovery, very large numbers of some types of complexes have now been reported, for example, cisplatin analogues for anticancer studies; however, the road from initial synthesis and testing to clinical trials remain long and torturous, and most complexes fail to progress beyond the original stages of synthesis, characterization and preliminary testing. In such areas, the need to publish means that authors all too rarely have the time to take a step back and consider the bigger picture; there is

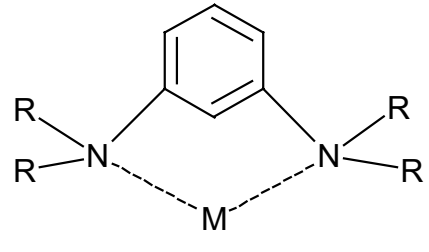

(1)

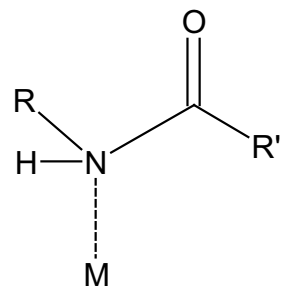

(2)

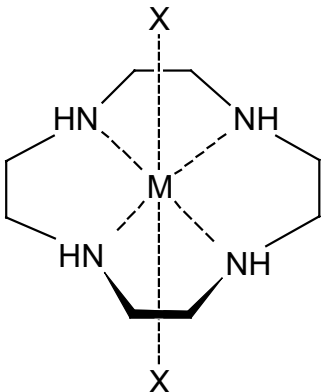

(3)
Scheme 1 Three problematic structures: (1) It is geometrically impossible for meta- substituents to coordinate to a single metal centre. (2) This amide N-donor has retained its proton. This is very unlikely, as the nitrogen lone pair is delocalized over the amide group and so not generally available for coordination. More likely alterna- tives would be deprotonation of the $\mathrm{N}$ and coordination as an anion, or coordination through the amide O. (3) This macrocyclic ligand is too small to coordinate a transition metal in the equatorial mode. The $\mathrm{X}$-groups would more likely be cis to each other 
too much pressure to simply move on to the next combination of metals and ligands. At TMC, this is manifested in the ever-increasing reluctance of authors to write insightful review articles of their specialist areas. This situation will not change until and unless those tasked with assessing research outputs come to recognize critical reviews as valid contributions in their own right.

As my own involvement with TMC draws to a close, I am delighted to welcome Martin Lemaire to the journal as Associate Editor. I am confident that the future of the journal will be in good hands. Looking further ahead, what will the next 30 years have in store for TMC? It is appropriate that the United Nations has designated this year as the International Year of the Periodic Table of Chemical Elements (IYPT 2019), in order to highlight the central importance of chemistry in the worldwide economy. Several aspects of transition metal chemistry are particularly relevant in this context. The goal of sustainable development will require ongoing research into catalytic processes for a wide range of industrial chemistry applications; ideally, these should be based on abundant and environmentally benign metals such as iron and copper. There is much work to be done in this context. In the energy sector, transition metals can be exploited in solar powered applications, including photovoltaics and photocatalysts, as well as in the development of more energy-efficient devices. In health, transition metal complexes already find a range of applications, including use as drugs and medical imaging agents; the natural occurrence of transition metals in many proteins such as superoxide dismutase and haemoglobin suggests that we would do well to take a more holistic approach to the application of transition metals in health and medicine. Beyond these considerations, I will make no predictions for the next 30 years of TMC, but I do have some aspirations. First, I hope that the long-term trend towards more substantial papers will continue. This has little to do with the artificial metrics of impact factors, much more to do with maximizing the value of the journal to our readers. I hope and expect to see whole new areas of transition metal chemistry emerge, both to fascinate those who appreciate the endless variety and subtlety of our discipline, and also to lead to novel and valuable real-world applications. Finally, we need to encourage future generations of scientists to find the time, perseverance, and sheer determination to chase down odd results, anomalies and curiosities, because this is the wellspring of all the best discoveries.

\section{Reference}

1. Groom CR, Bruno IJ, Lightfoot MP, Ward SC (2016) The Cambridge structural database. Acta Cryst B 72:171-179

Publisher's Note Springer Nature remains neutral with regard to jurisdictional claims in published maps and institutional affiliations. 